\title{
La incorporación de tecnologías emergentes en la formación de alumnos del área económico-administrativo
}

\section{The emerging technologies integrated into the training of students in the economic- administrative area}

\author{
SOBERANES-MARTÍN, Anabelem†*, CASTILLO-MENDOZA, José Luis, COTERA-REGALADO,
} Esperanza y MARTÍNEZ-REYES, Magally

Universidad Autónoma del Estado de México, Centro Universitario UAEM Valle de Chalco, Hermenegildo Galeana \#3, Col. María Isabel, Valle de Chalco Solidaridad, Estado de México. C. P. 56615

\author{
ID $1^{\text {er }}$ Autor: Anabelem, Soberanes-Martín / ORC ID: 0000-0002-1101-8279, CVU CONACYT ID: 180105 \\ ID $1^{\text {er }}$ Coautor: José Luis, Castillo-Mendoza / ORC ID: 0000-0002-5668-0602, CVU CONACYT ID: 898715 \\ ID $2^{\text {do }}$ Coautor: Esperanza, Cotera-Regalado / ORC ID: 0000-0002-2618-4245, CVU CONACYT ID: 450072 \\ ID $3^{\text {er }}$ Coautor: Magally, Martínez-Reyes / ORC ID: 0000-0002-2643-6748, CVU CONACYT ID: 202661
}

\begin{abstract}
Resumen
El presente trabajo está enfocado en proponer la incorporación de Tecnologías Emergentes (TE) en el área Económico Administrativo (AEA), específicamente para los estudiantes de la Licenciatura en Contaduría (LCN) y la Licenciatura en Informática Administrativa (LIA), del Centro Universitario Valle de Chalco de la UAEMex, debido a que éstos están enfocados al desarrollo y creación de empresas, iniciando con el uso de móviles, la realidad aumentada y apoyos virtuales. Se elaboró un instrumento con escala Likert, se aplicó a una muestra de 238 estudiantes de LIA y LCN, tomando el total de alumnos como población (408) y se realizó un muestreo por conveniencia. Además de una entrevista a 12 profesores de ambas licenciaturas. El enfoque considerado en la investigación fue de tipo cualitativo, transversal y de campo. La validación del cuestionario fue a través del alfa de Cronbach (0.81) y su calificación mediante la media y la desviación estándar, codificando cada uno de los ítems de acuerdo con las características de la población de estudio. Entre los resultados se logró que los docentes aceptaran incorporar las tecnologías emergentes y diseñar cómo las implementarán en el aula, para que mediante las estrategias propuestas se contribuya en el aprendizaje de los estudiantes del área económico-administrativo.
\end{abstract}

Nuevas tecnologías, Estrategia de aprendizaje, Enseñanza superior

\begin{abstract}
The present work is focused on proposing the incorporation of Emerging Technologies (ET) in the EconomicAdministrative area (EAA), specifically for students of the Bachelor's Degree in Accounting (LCN, Spanish acronym) and the Bachelor's Degree in Administrative Informatics (LIA, Spanish acronym), of the Chalco Valley University Center of the UAEMex, because they are focused on the development and creation of businesses, starting with the use of mobile phones, augmented reality and virtual supports. An instrument with a Likert scale elaborated, applied to a sample of 238 students of LIA and LCN, taking the total of students as population (408) and a convenience sampling was carried out. Also, there was an interview with 12 professors of both bachelor's degrees. The focus considered in the research was qualitative, transversal, and field. The questionnaire validated with Cronbach's alpha (0.81) and its score applying the mean and standard deviation, coding each of the items according to the characteristics of the study population. Among the results, teachers agreed to incorporate emerging technologies and to design how they will implement them in the classroom so that the proposed strategies contribute to the learning of students in the economic-administrative area.
\end{abstract}

New technologies, Learning strategy, Higher education

Citación: SOBERANES-MARTÍN, Anabelem, CASTILLO-MENDOZA, José Luis, COTERA-REGALADO, Esperanza y MARTÍNEZ-REYES, Magally. La incorporación de tecnologías emergentes en la formación de alumnos del área económicoadministrativo. Revista de Simulación Computacional. 2019. 3-10: 6-15

\footnotetext{
$\dagger$ Investigador contribuyendo como primer autor.
} 


\section{Introducción}

La globalización ha generado que desde hace varios años exista demanda en el campo laboral de personal mejor capacitado, aunado a lo expresado por Sokołowicz (2019) el incremento de estudiantes en la población urbana contribuye significativamente al potencial intelectual de las ciudades y regiones. En el caso del ambiente empresarial la calidad considerada como un producto final eficiente, se materializa en un profesionista que ejerza competencias laborales, con un sentido innovador.

Firsov, Firsova, \& Rabotnikova (2019) señalan que la aplicación de la innovación en las interacciones entre las instituciones educativas y los empleadores formará los requisitos necesarios para una respuesta a las condiciones cambiantes del campo laboral. Es decir, como lo expresan Tarango et al. (2019) se debe buscar cumplir con las expectativas definidas por los empleadores.

Para lograrlo, las instituciones educativas se van adaptando a las necesidades a través de combinar actividades realizadas tradicionalmente con incorporar otras, mediadas o no por tecnología, pero que involucra cambios en la actividad del profesor y del alumno. Pero, adoptar la tecnología en el aula de clase requiere profundizar en su significado, puesto que el profesor puede ocupar la tecnología y el alumno ser un espectador del resultado, o bien puede el alumno utilizar algún simulador o software en clase o fuera para contribuir en su aprendizaje y ser responsable de su aprendizaje, con o sin la guía del profesor.

Las universidades deben de prepararse para emplear las TE fomentando nuevas competencias, por lo tanto, se debe plantear la posibilidad de incorporar la tecnología como un elemento estratégico para lograr el éxito de los estudiantes (D2L, 2019). La Universidad Autónoma del Estado de México (UAEMéx) no es ajena a la situación planteada, motivo por el cual, en la restructuración de sus planes de estudio de 2018, señala "[...] educar a más personas con más calidad, mediante procesos y modelos de enseñanza basados en el uso de tecnologías de la información y comunicación, con personal académico altamente calificado e infraestructura adecuada para el desarrollo de las capacidades y habilidades de los alumnos." (UAEMéx, 2018a, p. 7; UAEMéx, 2018a, p. 9).
El objetivo del Programa Educativo (PE) de la Licenciatura en Informática Administrativa (LIA) es "[...] formar profesionales con conocimientos sólidos en Tecnologías de la Información que diseñen, innoven e implementen sistemas de información con el fin de aplicarlos a los procesos de planeación, organización, dirección y control de una organización [...]” (p. 131).

El plan de estudio está integrado por seis áreas curriculares: Administración, Contaduría y finanzas, Idiomas, Ingeniería y seguridad y Lenguajes y Sistemas, y Tecnología y arquitectura. Con 44 Unidades de Aprendizaje (UA) obligatorias y 3 optativas, además de 2 actividades académicas, con un total de 358 créditos. La distribución del número de UA por cada área curricular, además de los créditos se observa en la tabla 1.

\begin{tabular}{|l|r|r|}
\hline \multicolumn{1}{|c}{ Área curricular } & \multicolumn{2}{c|}{$\begin{array}{l}\text { Unidades de } \\
\text { aprendizaje }\end{array}$} \\
\hline Administración & 8 & 53 \\
\hline Contaduría y finanzas & 4 & 27 \\
\hline Idiomas & 4 & 24 \\
\hline Ingeniería y seguridad & $12+2 *$ & 117 \\
\hline Lenguajes y sistemas & 14 & 92 \\
\hline Tecnología y Arquitectura & 11 & 75 \\
\hline *Actividades académicas
\end{tabular}

Tabla 1 Resumen curricular del PE de LIA

Fuente: Elaboración Propia con información de (UAEMéx, 2018a, p. 155)

El porcentaje del número de UA por cada área curricular se presenta en la figura 1, en donde se puede identificar que dos áreas de estudio: administración y contaduría y finanzas, representan 24\% del plan de estudios. En el grafico 1 se muestran los porcentajes del número de UA por área curricular.

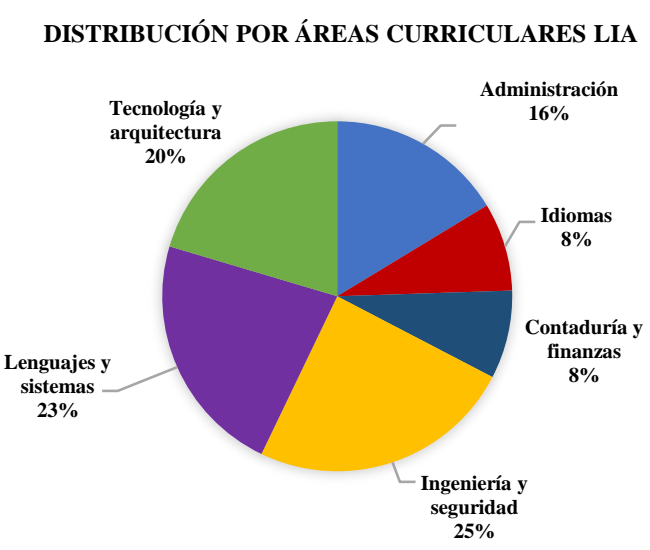

Grafico 1 Distribución del porcentaje de UA por área curricular de LIA

Fuente: Elaboración propia con información de UAEMex, $2018^{a}$

SOBERANES-MARTÍN, Anabelem, CASTILLO-MENDOZA, José Luis, COTERA-REGALADO, Esperanza y MARTÍNEZ-REYES, Magally. La incorporación de tecnologías emergentes en la formación de alumnos del área económico-administrativo. Revista de Simulación Computacional. 2019 
En relación con la Licenciatura en Contaduría (LCN) tiene como objetivo "[...] formar profesionales que generen, analicen e interpreten información financiera y administrativa, detecten y propongan soluciones a los problemas económicos y materiales de una organización y lograr la mejor toma de decisiones, con alto sentido de responsabilidad, de ética y de servicio [...]”' (p. 136).

Entre las competencias requeridas para el desempeño de las funciones y tareas, se considera el empleo de software y Tecnologías de la Información y Comunicación (TIC) aplicados a la contabilidad financiera. El plan de estudios también está integrado por seis áreas curriculares: Administración, Auditoría, Contaduría, Derecho, Economía y finanzas e Idiomas. Con 52 unidades de aprendizaje obligatorias y 5 optativas, además de 2 actividades académicas, para un total de 401 créditos (ver tabla 2).

\begin{tabular}{|l|r|r|}
\hline \multicolumn{1}{|c}{ Área curricular } & $\begin{array}{c}\text { Unidades de } \\
\text { aprendizaje }\end{array}$ & Créditos \\
\hline Administración & 9 & 57 \\
\hline Auditoría & 5 & 30 \\
\hline Contaduría & $19+2 *$ & 153 \\
\hline Derecho & 14 & 90 \\
\hline Economía y finanzas & 18 & 102 \\
\hline Idiomas & 4 & 24 \\
\hline *Actividades académicas & &
\end{tabular}

Tabla 2 Resumen curricular del PE de LIA.

Fuente: Elaboración Propia con información de (UAEMéx, 2018b, p. 168)

La distribución por áreas curriculares se organiza de manera diferente a LIA, para cuestiones de investigación se determinó con los profesores de las áreas de docencia considerar tres: economía y finanzas, administración y contaduría, lo que representa $47 \%$ del plan de estudios (ver grafico 2).

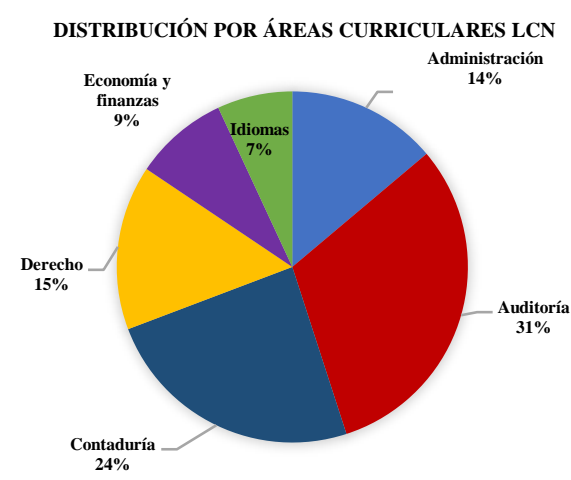

Grafico 2 Distribución del porcentaje de UA por área curricular de LCN

Fuente: Elaboración propia con información de UAEMéx, $2018 b$

ECORFAN® Todos los derechos reservado
Una vez que se ha identificado la importancia de las UA del AEA en la formación de LIA y LCN, se reconoce que los docentes emplean la tecnología como apoyo didáctico (para exponer, búsqueda de información, elaboración de material didáctico, alojar información, entre otros), se busca fomentar las TE para la práctica de la disciplina y más aún que sirvan para involucrar al estudiante en entornos de simulaciones reales del ejercicio de la profesión.

Para lograrlo se necesita identificar cómo se va implementar, para ello hace falta considerar las estrategias de aprendizaje, entendidas como: "[...] procesos que conducen a la toma de decisiones -conscientes e intencionales-; también conllevan a seleccionar los conocimientos -bien sean conceptuales, procedimentales y/o actitudinales- [...]" (León, Risco y Alarcón, 2014, p. 129).

Sin embargo, se debe considerar lo expresado por Mata-López \& Tobón (2019) sobre los desafíos tecnológicos de los profesores: 1) Preparar y mantener un equipo de docentes tecnológicos competentes, 2) Planificar y organizar procesos para definir proyectos, 3 ) Identificar sistemáticamente los requisitos de la integración de la tecnología educativa, 4) Redefinir y refinar los roles de los maestros, 5) Gestionar los recursos, además de proporcionar mantenimiento y soporte (p. 5).

Asimismo, Baroudi \& Rodjan (2019) expresan que existen limitantes como recursos limitados en TIC y laboratorios y que los maestros no están capacitados en TIC. De igual manera, León, Risco y Alarcón (2014) indican: "[...] la infraestructura institucional implementada conlleva al éxito del propósito, siempre y cuando se genere un ambiente propicio para que los estudiantes adquieran las competencias cognitivas, actitudinales, procedimentales y sociales que les ayuden a integrar a sus estructuras." (p. 141).

De acuerdo con Pozo y Postigo (1993, citados por Visbal-Cadavid, Mendoza-Mendoza, \& Díaz, 2017) señalan que la aplicación de estrategias no es automática sino controlada, que se debe precisar la planificación y control de la ejecución y están relacionadas con la metacognición o conocimiento sobre los propios procesos mentales, además implican un uso selectivo de los propios recursos y capacidades disponibles.

SOBERANES-MARTÍN, Anabelem, CASTILLO-MENDOZA, José Luis, COTERA-REGALADO, Esperanza y MARTÍNEZ-REYES, Magally. La incorporación de tecnologías emergentes en la formación de alumnos del área económico-administrativo. Revista de Simulación Computacional. 2019 
Montes de Oca \& Machado (2011) definen la estrategia docente como la secuencia de acciones que "[...] atendiendo a todos los componentes del proceso, guían la selección de los métodos y recursos didácticos más apropiados para la dirección del aprendizaje, teniendo en cuenta las condiciones en que éste transcurre, la diversidad de los estudiantes, contenidos y procesos para alcanzar los fines educativos propuestos" (p. 483).

Un factor determinante para un buen aprendizaje y desarrollo académico óptimo es el uso de estrategias de aprendizaje (VisbalCadavid, Mendoza-Mendoza, \& Díaz, 2017, p. 73). De ahí, que la investigación haga referencia a estrategias de aprendizaje para incorporar TE en el AEA, específicamente para los estudiantes de LCN y LIA, considerando las condiciones del espacio académico en cuestión, en cuanto a infraestructura, características de los docentes y objetivo de las áreas curriculares seleccionadas, para fomentar competencias para el desarrollo y creación de empresas, pero con el uso de los móviles, la realidad aumentada (RA) y apoyos virtuales (AV) en el proceso educativo.

\section{Metodología}

Para lograr el objetivo se realizo el diseño de la investigación.

\section{Diseño}

El enfoque que siguió la investigación fue de tipo cualitativa, transversal y de campo. Se trata de un estudio mediante una entrevista cualitativa comparativa y un cuestionario diseñado para ayudar a explorar el uso de tecnología emergente en el proceso de enseñanza y aprendizaje de los estudiantes de LIA y LCN en asignaturas del AEA. Los métodos cualitativos permiten analizar y comparar los datos en busca de similitudes o diferencias en los puntos de vista de los profesores y los alumnos sobre el tema y desarrollar propuestas.

\section{Participantes}

El muestreo intencional fue el método utilizado para el presente estudio. Con relación a los alumnos la población de LIA era de 140 y de LCN de 268, el tamaño de la muestra se calculó aplicando la propuesta de Fernández (2010), se utilizó la estimación de una proporción para un estudio de corte transversal con la fórmula:

$$
n=\frac{N * Z_{a}^{2} p * q}{d^{2} *(N-1)+Z_{a}^{2} * p * q}
$$

Donde:

$\mathrm{N}=$ Total de la población

$Z_{a}^{2}=1.962$ (la seguridad es del $95 \%$ )

$\mathrm{p}=$ proporción esperada $(5 \%=0.05)$

$\mathrm{q}=1-\mathrm{p}(1-0.05=0.95)$

$\mathrm{d}=$ precisión $($ se desea $3 \%)$

Se determinaron las muestras de alumnos obteniéndose 96 y 142 alumnos para LIA y LCN respectivamente, la figura 1 presenta el porcentaje por género de alumnos participantes. Se les invitó a través de las coordinaciones de licenciatura, los alumnos en promedio tienen 21.5 años.
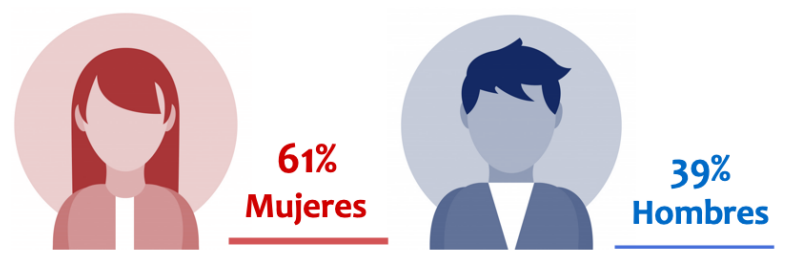

Figura 1 Participación de alumnos por género Fuente: Elaboración Propia

Con relación a los docentes, se les convocó a reunión, ahí se les invitó a participar en el estudio. Doce profesores aceptaron participar ( $\mathrm{n}=8$ de LCN y $\mathrm{n}=4 \mathrm{LIA})$. El número de participantes fue satisfactorio porque participaron el total de profesores que imparten UA de las áreas curriculares seleccionadas durante el semestre 2019A (febrero-julio). Tres hombres con edad promedio de 46 años y 9 mujeres con edad promedio de 39 años, de los cuales tres tienen estudios de licenciatura, seis tiene maestría y tres con doctorado. Cabe mencionar que cuatro profesoras participan en ambas licenciaturas.

\section{Procedimiento}

El estudio se realizó durante el segundo trimestre del semestre 2019A. Se les aplicó a los profesores una entrevista cara a cara (en promedio se tardaron en contestar 40 minutos). Posteriormente, se les aplicó a los alumnos un cuestionario diagnóstico, que contestaron en línea en las salas de cómputo de la institución, sin ningún docente presente. 
Enseguida, se realizaron cuatro reuniones de trabajo, todas las actividades se realizaron en el Centro Universitario Valle de Chalco de la UAEMex. Las reuniones duraron en promedio una hora y 30 minutos, en ellas, se acordaron las TE a utilizar y la forma de incorporarlas (guías pedagógicas).

\section{Herramientas de recopilación de datos}

La entrevista semiestructurada para los docentes está integrada por 15 preguntas, las cuales fueron grabadas, los participantes señalan sus experiencias y prácticas, las preguntas fueron diseñadas para identificar los siguientes aspectos: a) qué tecnologías utilizan, b) cómo utilizan las tecnologías en el aula, c) cómo podrían implementar las TE en las clases, d) qué dificultades podrían experimentar con la implementación, e) qué habilidades tienen en relación con el uso de las TIC y, f) la disponibilidad en la institución de recursos en materia de TIC.

El cuestionario para los alumnos está integrado con 20 preguntas cerradas en escala Likert, se empleó la herramienta LimeSurvey para la recolección de información, la validación del instrumento se realiza por medio de una alfa de Cronbach (0.81) y su calificación por estadísticos como la media y la desviación estándar, codificando cada uno de los ítems de acuerdo con las características de la población de estudio.

\section{Resultados}

La información obtenida por los docentes se obtuvo al aplicarles una entrevista semiestructurada, se codificaron las respuestas, a continuación se presentan algunos de los resultados en cinco aspectos:

Sobre las tecnologías, los docentes reportan que principalmente utilizan el proyector y equipo de cómputo para sus clases, seguido de los laboratorios o salas de cómputo, el mismo número de profesores (4) emplean internet y hacen uso de software especializado, finalmente, tres docentes emplean plataformas educativas, en la tabla 3 se presenta la información con porcentaje de frecuencia de cada una.

\begin{tabular}{|l|c|}
\hline \multicolumn{1}{|c|}{ Tecnología } & \% \\
\hline Proyector & 73.3 \\
\hline Laptop para el proyector & 73.3 \\
\hline Computadoras/laboratorios & 58.3 \\
\hline Internet en clase & 33.3 \\
\hline Software especializado & 33.3 \\
\hline $\begin{array}{l}\text { Manejo de plataformas (Moodle, Schoology, } \\
\text { Seduca) }\end{array}$ & 8.3 \\
\hline
\end{tabular}

Tabla 3 Tecnologías utilizadas por los docentes del AEA. Fuente: Elaboración Propia

Con relación a la manera que los profesores utilizan las tecnologías en el aula, entre las respuestas más frecuentes los doce profesores hacen uso de las TIC para apoyar la exposición de los temas al igual que para buscar información, nueve emplean redes sociales o repositorio de alojamiento de archivos (Dropbox y Google Drive principalmente) y correo electrónico; cuatro para ejercitar temas, tres se apoyan con herramientas como Schoology o Mimio para evaluar. Hay que resaltar que ningún docente utiliza alguna de las tecnologías bajo estudio, la tabla 4 muestra los usos más frecuentes y los porcentajes correspondientes.

\begin{tabular}{|l|l|}
\multicolumn{1}{|c|}{ Uso Actual } & \% \\
\hline Presentaciones/exposiciones & 100 \\
\hline Buscar información & 100 \\
\hline $\begin{array}{l}\text { Internet (redes sociales, alojamiento de } \\
\text { archivos, correo electrónico) }\end{array}$ & 75.0 \\
\hline Ejercitar/aplicar temas & 33.3 \\
\hline Para evaluación & 25.0 \\
\hline Tecnologías emergentes (RA, Móviles, AV) & 0 \\
\hline
\end{tabular}

Tabla 4 Maneras de utilizar actualmente las tecnologías por los docentes del AEA

Fuente: Elaboración Propia

De la manera en que se podrían implementar las TE en las clases, en la tabla 5 se exponen las aportaciones de los docentes sobre el tópico, en donde resalta el uso para aplicar los temas y que el docente demuestre el tema, seguido de para ejercitar el tema con $75 \%$, cabe rescatar que para evaluación solo $25 \%$ lo considera.

\begin{tabular}{|l|c|}
\hline \multicolumn{1}{|c|}{ Propuesta } & \% \\
\hline Demostración de la temática & 91.6 \\
\hline Aplicar el tema & 91.6 \\
\hline Para ejercitar el tema & 75.0 \\
\hline Prepararse sobre el tema & 33.3 \\
\hline Para repaso & 25.0 \\
\hline Para evaluación & 25.0 \\
\hline
\end{tabular}

Tabla 5 Propuestas de implementación Fuente: Elaboración Propia 
Existen dificultades que se podrían experimentar para llevar a cabo la implementación de TE en el aula, entre ellas se identifican principalmente, con $91.6 \%$ aceptación de los actores involucrados en el proceso de aprendizaje y capacidad en laboratorios, con $83.3 \%$ el tiempo necesario para preparar la implementación, capacitación de los docente o alumnos si es necesario, selección de tecnologías a utilizar y diseño instruccional para su empleo, con $75 \%$ expresan los profesores contar con equipo suficiente, principalmente lo señalan para los laboratorios en donde en promedio se tienen 23 equipos para grupos de hasta 45 alumnos (ver tabla 6).

\begin{tabular}{|l|c|}
\hline \multicolumn{1}{|c|}{ Dificultades } & $\%$ \\
\hline Capacidad de laboratorios & 91.6 \\
\hline Aceptación de directivos, docentes o alumnos & 91.6 \\
\hline $\begin{array}{l}\text { Tiempo de preparación (capacitación, diseño } \\
\text { instruccional) }\end{array}$ & 83.3 \\
\hline Equipo insuficiente & 75.0 \\
\hline Falta de capacitación docente & 58.3 \\
\hline
\end{tabular}

Tabla 6 Posibles dificultades para incorporar TE. Fuente: Elaboración Propia

Las habilidades sobre el uso de las TIC, $100 \%$ de los docentes consideran que cuentan con buen nivel de conocimientos, además manejan paquetes de ofimática, de igual manera tienen habilidad para manejar software especializado del área y en menor porcentaje algunos manejan plataformas de gestión (tabla 7).

\begin{tabular}{|l|c|}
\hline \multicolumn{1}{|c|}{ Habilidades } & $\%$ \\
\hline Buen nivel de conocimientos & 100 \\
\hline Manejo de ofimática & 73.3 \\
\hline Operar dispositivos móviles & 66.6 \\
\hline $\begin{array}{l}\text { Manejo de software especializado de la } \\
\text { disciplina }\end{array}$ & 33.3 \\
\hline $\begin{array}{l}\text { Manejo de plataformas (Moodle, Schoology, } \\
\text { Seduca) }\end{array}$ & 16.6 \\
\hline
\end{tabular}

Tabla 7 Habilidades docentes sobre TIC Fuente: Elaboración Propia

La tabla 8 muestra la disponibilidad de recursos en materia de TIC que consideran podrían utilizar en sus asignaturas, principalmente consideran que aplicaciones para móviles, seguido de software para utilizar en los laboratorios y con el mismo porcentaje simulares de negocios y apoyos virtuales, en este último aspecto se refirieron a aplicaciones con realidad aumentada o virtual.

\begin{tabular}{|l|c|}
\hline \multicolumn{1}{|c|}{ Recursos } & $\%$ \\
\hline Aplicaciones para móviles & 75.0 \\
\hline Software para computadora & 33.3 \\
\hline Simuladores & 16.6 \\
\hline Apoyo virtual & 16.6 \\
\hline
\end{tabular}

Tabla 8 Disponibilidad de TE para las asignaturas del AEA

Fuente: Elaboración Propia

De los resultados del cuestionario aplicado a los estudiantes, a continuación, se presentan algunos resultados:

Los recursos tecnológicos que los estudiantes conocen y han manejado con fines educativos, $100 \%$ refieren el smartphone considerando que lo utilizan para entregar actividades o comunicarse con los compañeros o docentes, en redes sociales, búsqueda de información en internet y reproducir videos para reforzar algún tema, con relación a aplicaciones de realidad aumentada o virtual 51 alumnos los han utilizado (todos fueron de LIA) y software especializado 145 reportaron que lo han utilizado (96 de LIA y el resto de LCN), en cuestiones relacionadas con el AEA, Excel y paquetería de ASPEL, la figura 2 muestra los porcentajes del diagnóstico.

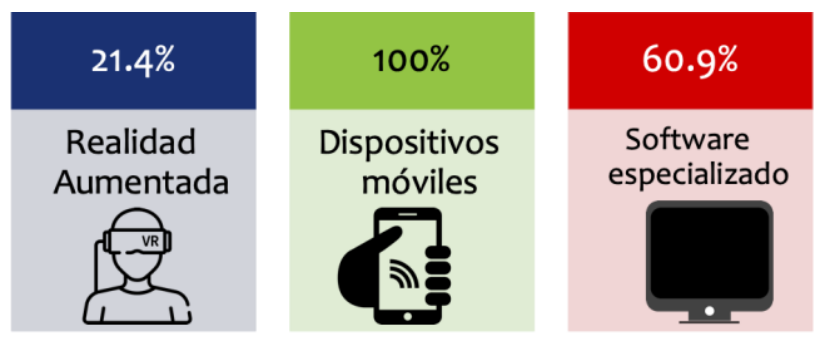

Figura 2 Resultados de diagnóstico de uso de tecnología Fuente: Elaboración propia

Se les pregunto a los alumnos sobre el tiempo promedio que pasan al día utilizando algún dispositivo tecnológico (teléfono celular, tableta, laptop, computadora, videojuego), el menor porcentaje lo tienen los dos extremos de los rangos presentados, menos de 2 hrs. y que más de 5 , después con $27.8 \%$ se encuentran aquellos que pasan de 3 a 5 hrs. y con mayor frecuencia quienes pasan entre 2 y 3 hrs. con $47.9 \%$. 


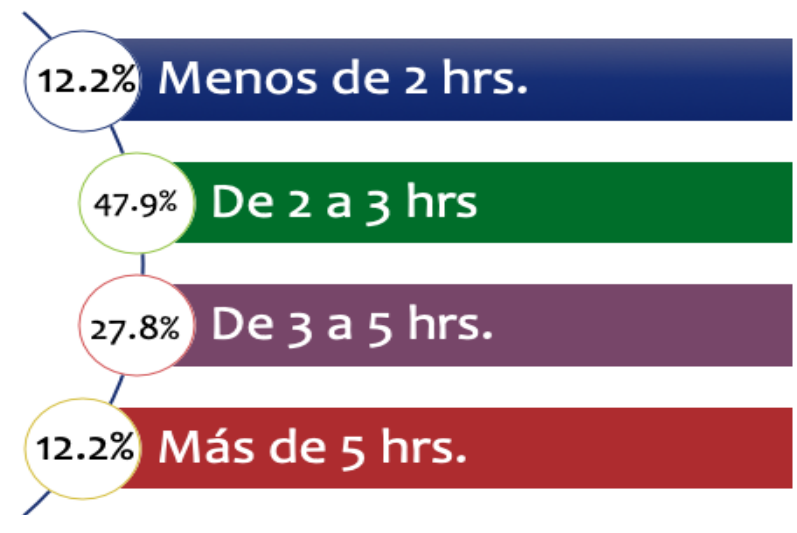

Figura 3 Tiempo que utilizan los alumnos algún dispositivo tecnológico

Fuente: Elaboración propia

Con qué frecuencia utilizan los profesores de las asignaturas del AEA tecnología digital en sus clases, los alumnos refieren $10.9 \%$ que siempre, $26.9 \%$ casi siempre, con mayor porcentaje que algunas veces y con casi el $15 \%$ que casi nunca y nunca; lo que permitió identificar que los docentes tratan de emplear la TIC en el proceso de enseñanza y que cuentan con habilidades para poder utilizarlas.

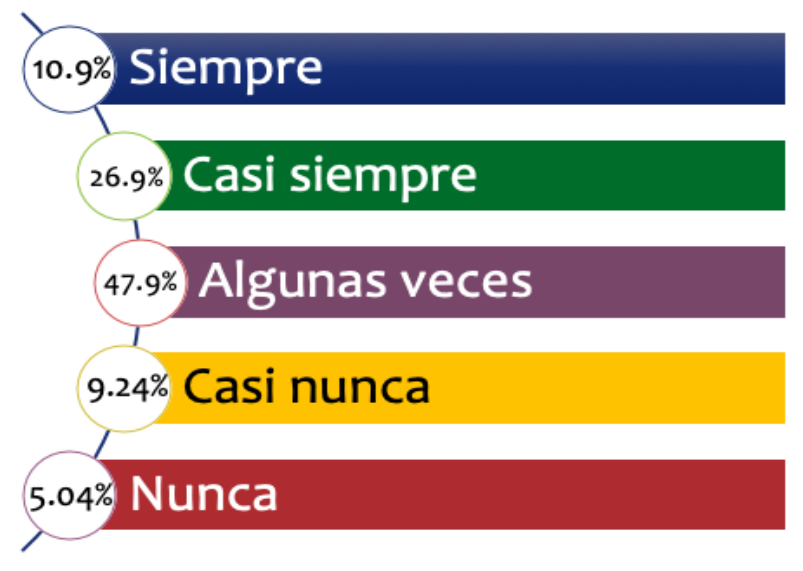

Figura 4 Frecuencia de uso de TIC en el aula Fuente: Elaboración Propia

Sobre si les gustaría utilizar recursos tecnológicos en asignaturas del AEA, los estudiantes respondieron $30.67 \%$ que mucho, $47.9 \%$ que bastante y $21.42 \%$ que regular, se debe resaltar que nadie contesto que poco o nada, lo que permite identificar la importancia que consideran los alumnos de las TIC para su aprendizaje (figura 5).
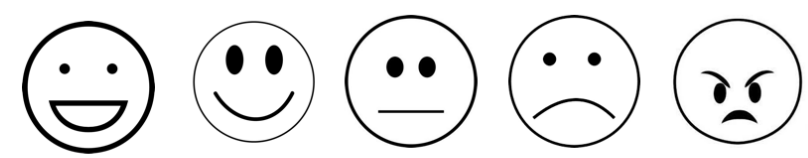

$30.67 \%$

$47.90 \%$

$0 \%$

$0 \%$
Regular

Figura 5 Resultados de diagnóstico

Fuente: Elaboración Propia

Si les gustaría a los alumnos utilizar aplicaciones móviles para algunos temas en sus clases del AEA, casi el 90\% señalo que estaría de acuerdo o muy de acuerdo, esto tiene relación con el porcentaje del uso de dispositivo móvil en la vida cotidiana (ver figura 6 ).

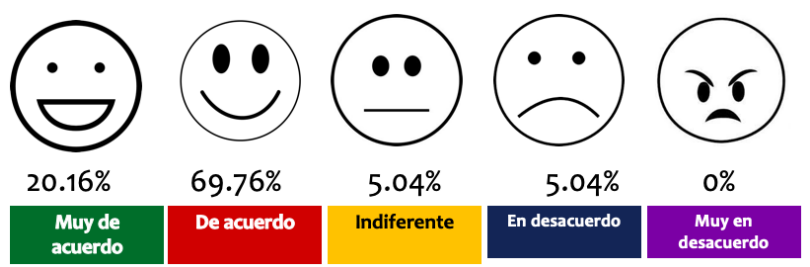

Figura 6 Interés por utilizar aplicaciones móviles en el aula

Fuente: Elaboración Propia

Los estudiantes respondieron en relación con que les gustaría emplear aplicaciones con realidad aumentada para algunos temas en sus clases del AEA, la mayoría (66.38\%) estarían de acuerdo, mientras que al $15.16 \%$ le es indiferente, $8.38 \%$ esta muy de acuerdo (ver figura 8).

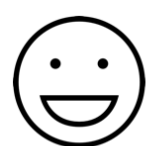

$8.38 \%$

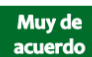

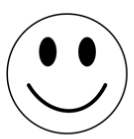

$66.38 \%$

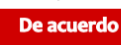

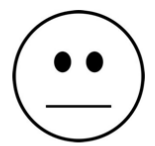

$15.16 \%$

Indiferente
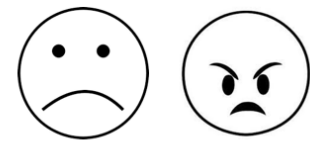

$5.04 \%$

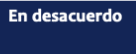

Figura 7 Interés por emplear aplicaciones con RA Fuente: Elaboración Propia

Los dicentes les atraería que se incorporarán simuladores, por ejemplo, de negocios en algunas materias del AEA, la figura 8 presenta los porcentajes en donde se identifica que casi $80 \%$ contestaron que están de acuerdo o muy de acuerdo, lo cual permite identificar como un área de oportunidad.

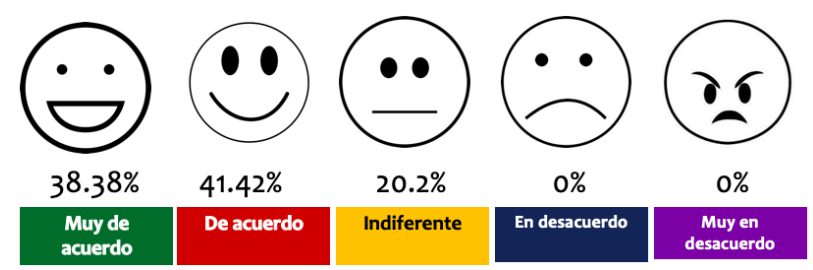

Figura 8 Interés por usar simuladores Fuente: Elaboración Propia 
Los resultados demuestran que no sería complejo su incorporación, al no ser una actividad nueva el uso de tecnología en el aula, desde 2012 se ha incorporado el uso de recursos educativos para alumnos de LIA, sin embargo, ha sido para asignaturas de cálculo, estadística, sistemas de información entre otras (Soberanes, Castillo \& Peña, 2017), o en su caso simuladores para materias relacionadas con redes (comunicación entre computadoras 1 y 2, sistemas operativos para red, entre otras). Pero, para el AEA no, de ahí lo importante de la propuesta.

Con la información obtenida por los dos instrumentos diagnósticos, permitieron trabajar en la planeación para incorporar las TE en las clases de los alumnos de LCN y LIA.

Primero, al identificar que todos los estudiantes han utilizados dispositivos móviles se planteo el plan de acción para incorporarse durante el semestre 2019B (agosto 2019- enero 2020), en las reuniones de trabajo se realizaron principalmente cuatro actividades:

Se inició con el análisis de contenido para su tratamiento didáctico, se realizó un documento por cada una de las cuatro asignaturas seleccionadas, se tenia que identificar en términos de porcentajes el tipo de contenidos por unidad temática, para identificar en que tema se requería utilizar la aplicación. Los tipos de contenidos se indicaron en conceptuales, procedimentales y actitudinales, en la tabla 9 se presenta un ejemplo del análisis de contenidos de una asignatura del AEA.

\begin{tabular}{|c|c|c|c|}
\hline Tipo de Contenidos & Conceptuales & Procedimentales & Actitudinales \\
\hline $\begin{array}{ll}\text { Estilos } & \text { de } \\
\text { negociación } & \end{array}$ & $55 \%$ & $20 \%$ & $25 \%$ \\
\hline $\begin{array}{l}\text { Planificación } \\
\text { negociación }\end{array}$ & $65 \%$ & $20 \%$ & $15 \%$ \\
\hline $\begin{array}{ll}\begin{array}{l}\text { Estilo } \\
\text { negociación }\end{array} & \text { de } \\
\end{array}$ & $65 \%$ & $20 \%$ & $15 \%$ \\
\hline $\begin{array}{l}\text { Habilidades de } \\
\text { negociar }\end{array}$ & $65 \%$ & $10 \%$ & $25 \%$ \\
\hline
\end{tabular}

Tabla 9 Análisis de contenido

Fuente: Elaboración Propia

Después de identificar los contenidos se debían seleccionar las aplicaciones para abordar los temas seleccionados, se revisaron diversas aplicaciones (aproximadamente 18) y determino utilizar en está primera fase 4 aplicaciones para móviles, las cuales son:

\begin{tabular}{|l|l|}
\hline \multicolumn{1}{|c|}{ Aplicación } & \multicolumn{1}{|c|}{ Asignatura } \\
\hline $\begin{array}{l}\text { XPlanner Gestión de } \\
\text { proyectos }\end{array}$ & Gestión de proyectos \\
\hline Sprints-Proyectos ágiles & $\begin{array}{l}\text { Proyectos } \\
\text { inversión }\end{array}$ \\
\hline Inventario para empresas & Contabilidad \\
\hline $\begin{array}{l}\text { Monetiza tu negocio en 6 } \\
\text { pasos }\end{array}$ & $\begin{array}{l}\text { Desarrollo } \\
\text { empresarial }\end{array}$ \\
\hline
\end{tabular}

Tabla 9 Aplicaciones y asignaturas a incorporar Fuente: Elaboración Propia

En un tercer momento, se diseñaron las planeaciones didácticas las cuales incluyen como datos de identificación: el nombre de la licenciatura, el titulo de la unidad de aprendizaje, el titulo de la unidad temática, el semestre al que corresponde, el objetivo de la unidad, número de sesiones, duración/horas, horas teóricas, horas prácticas. En el desarrollo de la secuencia didáctica se indican los contenidos de la actividad de enseñanza aprendizaje, las actividades por evaluar; materiales, medios y recursos, tiempo y escenario (estos aspectos en tres fases de inicio, desarrollo y cierre).

Después, se realizó la guía pedagógica de las cinco asignaturas, las cuales se componen de los elementos que se presentan en la tabla 10 , de acuerdo con los lineamientos que indica el artículo 87 del Reglamento de Estudios vigente, la guía pedagógica "[...] proporcionará recomendaciones para la conducción del proceso de enseñanza aprendizaje. Su carácter indicativo otorgará autonomía al personal académico para la selección y empleo de los métodos, estrategias y recursos educativos que considere más apropiados para el logro de los objetivos." (UAEMéx, 2018c, p. 34).

\begin{tabular}{|l|}
\hline \multicolumn{2}{|c|}{ Elementos } \\
\hline Datos de identificación \\
\hline Presentación de la guía pedagógica \\
\hline $\begin{array}{l}\text { Ubicación de la unidad de aprendizaje en el mapa } \\
\text { curricular }\end{array}$ \\
\hline Objetivos de la formación profesional \\
\hline Objetivos de la unidad de aprendizaje \\
\hline $\begin{array}{l}\text { Contenidos de la unidad de aprendizaje, y su } \\
\text { organización }\end{array}$ \\
\hline Acervo bibliográfico \\
\hline Mapa curricular \\
\hline
\end{tabular}

Tabla 10 Elementos que integran la guía didáctica Fuente: Elaboración Propia

La siguiente actividad fue la puesta en marcha de la propuesta en el aula, se esta realizando durante el semestre 2019B (agosto 2019-enero 2020). 


\section{Conclusiones}

Se identifica la importancia del perfil con lo que se ratifica lo expresado por Mata-López \& Tobón (2019) "es muy recomendable que el perfil del profesor se adapte a las necesidades académicas de los alumnos." (p. 13)

De igual manera, es importante que los programas educativos tengan en cuenta las necesidades de los empleadores (Firsov, Firsova, \& Rabotnikova, 2019).

La participación de los docentes en el desarrollo del proyecto se ratifica lo expresado por Montes de Oca \& Machado (2011) "Como parte de la estrategia docente deben elaborarse recursos didácticos que permiten proporcionar información, motivar a los estudiantes, guiar los aprendizajes, desarrollar habilidades, evaluar los conocimientos y habilidades y proporcionar espacios para la expresión y creación” (p. 487).

Se puede lograr el cumplimiento de los objetivos para incorporar las TE en la formación de LIA y LCN, el compromiso y motivación los docentes es de suma importancia para el logro del proceso de enseñanza aprendizaje; con el invaluable apoyo de los docentes demostraron estar comprometidos con el proyecto aunado al optimo nivel del uso de la tecnología y disponibilidad de tiempo, así como actitudes positivas y proactivas hacia las TIC (Flores, López \& Rodríguez, 2016), es decir, al sentirse involucrado y participar activamente en el diseño contribuye a reducir la renuencia (Baroudi \& Rodjan, 2019).

Como trabajo futuro, se establecieron los requerimientos para el desarrollo de una aplicación móvil con realidad aumentada (que será desarrollada con participación de alumnos de LIA) para la asignatura de Administración de las Pequeñas y Medianas Empresas (PyME), además se acordó que los docentes revisarían simuladores existentes para que en el período intersemestral (enero 2020) se tome la decisión de cuál utilizar y realizar dos diseños instruccionales, así como los instrumentos para evaluar la calidad de incorporar la tecnología, para que ambas tecnologías sean incorporadas durante el semestre 2020A (febrero-julio 2020).

\section{Referencias}

Baroudi, S. \& Rodjan, M. (2019). Behind the scenes: teachers' perspectives on factors affecting the implementation of inquiry-based science instruction. Research in Science \& Technological Education, 1-22. DOI: 10.1080/02635143.2019.1651259.

D2L. (2019). El futuro del trabajo y el aprendizaje en la era de la Cuarta Transformación Industrial. D2L Corporation. Recuperado de: https://www.d2l.com/es/futurodel-trabajo/

Fernández, P. (2010). Determinación del tamaño muestral. Fisterra. Disponible en: https://www.fisterra.com/mbe/investiga/9muest ras/9muestras 2.asp

Flores, K., López, M.C. \& Rodríguez, M.A. (2016). Evaluación de componentes de los cursos en línea desde la perspectiva del estudiante. Revista electrónica de investigación educativa, 18(1): 23-38. Disponible en: 2019 http://www.scielo.org.mx/scielo.php?script=sci _arttext\&pid=S1607-

$40412016000100002 \& \operatorname{lng}=$ es\&tlng=es

Firsov, Y., Firsova, I., \& Rabotnikova, N. (2019, May). Social partnership of employer and educational organizations as leadership innovations in training for small business. In 3rd International Conference on Social, Economic, and Academic Leadership (ICSEAL 2019). Atlantis Press.

León, A.P., Risco, E. \& Alarcón, C. (2014). Estrategias de aprendizaje en educación superior en un modelo curricular por competencias. Revista de la Educación Superior, 4(172): 123144.

Mata-López, W., \& Tobón, S. (2019). Analysis of Factors Associated to the Enrollment and Demand of Computing-Related Careers. Social Sciences, 8(1): 1-18.

Montes de Oca, N. \& Machado, E. (2011). Estrategias docentes y métodos de enseñanzaaprendizaje en la Educación Superior. Humanidades Médicas. 11: 475-488. 
Soberanes-Martín, A., Castillo-Mendoza, J.L., \& Peña-Martín, A. (2017). Uso de recursos educativos abiertos. Unviersidad\&Ciencia, 6(CIVITEC): 123-135.

Sokołowicz, M. E. (2019). Student cities or cities of graduates? The case of Lodz and its students declared preferences. Population, Space and Place, 25(2): e2177.

Tarango, L.E., Rodriguez-Morachis, M.A., Frausto, Y., de Jesus Rojas, E., \& Gaytán, M.L. (2019). Organizational Development in Improving Operations of a Language Center: Impact on Development of Students. In Advanced Macroergonomics and Sociotechnical Approaches for Optimal Organizational Performance (pp. 203-230). IGI Global.

UAEMéx. (2018a). Plan de estudios de Informática Administrativa. Toluca, México: UAEMex.

UAEMéx, (2018b). Plan de estudios de Contaduría. Toluca, México: UAEMex.

UAEMéx, (2018c). Reglamento de Estudios Profesionales. Toluca, México: UAEMex.

Visbal-Cadavid, D., Mendoza-Mendoza, A. \& Díaz, S. (2017). Estrategias de aprendizaje en la educación superior. Sophia, 13(2): 70-81. Disponible en: http://www.redalyc.org/pdf/4137/41375184400 8.pdf 